\title{
Gut Micobiota Alteration by Lactobacillus Rhamnosus Reduces Pro-inflammatory Cytokines and Glucose Level in the Adult Model of Zebrafish
}

\section{Fatemeh Bootorabi}

Tehran University of Medical Sciences, EMRIcal Science

\section{Farshid Saadat}

Guilan University of Medical Sciences

\section{Reza Falak}

Iran University of Medical Sciences

Hamed Manouchehri

Islamic Azad University Babol Branch

\section{Reza Changizi}

Islamic Azad University Babol Branch

\section{Hasan Mohammadi}

Tehran University of Medical Sciences

\section{Farnaz Safavifar}

Tehran University of Medical Sciences

\section{M.Reza Khorramizadeh ( $\nabla$ khoramza@tums.ac.ir)}

Tehran University of Medical Sciences Endocrinology and Metabolism Research Institute https://orcid.org/0000-0003-2563-8468

\section{Research note}

Keywords: Cytokine, Lactobacillus rhamnosus, probiotic, type 2 diabetes mellitus (T2DM), zebrafish

Posted Date: May 13th, 2021

DOl: https://doi.org/10.21203/rs.3.rs-505931/v1

License: (c) (i) This work is licensed under a Creative Commons Attribution 4.0 International License. Read Full License

Version of Record: A version of this preprint was published at BMC Research Notes on August 9th, 2021. See the published version at https://doi.org/10.1186/s13104-021-05706-5. 


\section{Abstract}

Objective: Type 2 diabetes mellitus (T2DM) is still a challenge for physicians to manage patient's circumstances. It is assumed that alterations in the normal flora may be involved in the pathogenesis of T2DM through inducing chronic inflammation. To investigate the effect of Lactobacillus rhamnosus as a common probiotic on T2DM, we induced an experimental model of T2DM in adult male Zebrafish by gradient hyper-glucose accumulation methodology.

Results: In this trial three-month old mail adult Zebrafish were divided in to four groups including two control groups and T2DM induced groups with or without probiotic treatment. After five days of acclimation, T2DM was induced by a gradient hyper-glucose accumulation methodology. Diabetic fishes had statistically abnormal blood glucose and pro-inflammatory cytokine levels compared to control group $(p=0.0001)$. This results suggest that probiotic intervention hindered the blood glucose level in the T2DM group by decreasing pro-inflammatory cytokines responsible for signaling in T2DM therapeutic modalities.

\section{Introduction}

Metabolic syndrome has steadily increased globally and many etiological factors including altered inflammatory states, adipose abnormalities and insulin resistance, contribute to their pathogenesis and thus should be considered in related basic studies [1-3]. Diabetes mellitus (DM) which is known by glycemic disturbances is usually categorized to various categories. Type $1 \mathrm{DM}$ patients are characterized by destruction of insulin-producing pancreatic beta-cells and type 2 diabetes mellitus (T2DM) patients with lowered response to insulin. The individual lifestyle and genetic code are usually co-risked in T2DM $[4,5]$. In obese T2DM patients, it was shown that losing weight reduces the glucose-lowering condition and can also improve glycemic control $[6,7]$. However, there are still multiple challenges for physicians to manage patients' condition, including side effects and toxicity of current therapeutics and medication failures [8]. Recently, several studies have reported that both T2DM and obesity are associated with gut microbiota composition $[1,9]$. Furthermore, nowadays it is useful to realize the effects of probiotics on management diabetes.

Probiotics are defined as live microorganisms with healthy beneficial to host if up taken in adequate amounts $[10,11]$. Recent advances have highlighted the beneficial outcome of gastrointestinal bacteria in the pathology of inflammatory disorders [12]. Much evidence suggests that probiotic uptake has beneficial effects on blood glucose through different mechanisms [13-16]. Since intestinal microbiota and their metabolites directly contribute in insulin resistance; probiotic could have a strong effect on physiological function by altering gastrointestinal bacterial community $[17,18]$. Consumption of a sufficient amount of probiotic is considered a therapeutic method for weight reduction and control of T2DM [19]. Furthermore, there is a direct correlation between the anti-diabetic drugs and gut microbiome community [9] . 
T2DM and obesity in the context of epidemiological data provide a solid platform for in vivo investigation of the disease. Nowadays, zebrafish (Danio rerio) has emerged as a powerful tool for scientific community among vertebrates. In the beginning of embryogenesis, zebrafish has transparent embryos with more than $85 \%$ of genetic similarity with human activity and thus as an attractive model organism for biomedical research [20]. Besides, it is also widely used for investigations on probiotics and host interaction with different gut microbiota and infectious diseases [21, 22].

Here in, we have developed a diet-induced zebrafish model for diabetes, to investigate the potential effect of oral administration of probiotic Lactobacillus rhamnosus ATCC ${ }^{\circledR} 53103^{\mathrm{Tm}}$ as a diet supplement for inflammation caused by T2DM. If probiotic intervention could hinder the blood glucose elevation in the T2DM model via impact on cytokine levels, it might be considered a novel therapeutic approach in T2DM treatment.

\section{Materials And Methods}

\section{Animals}

Adult Danio rerio AB strain was primarily received as a gift from Zebrafish core facility, University of Tampere, Finland. Fish were acclimated and subjected to cycles of breeding onward, under standard recirculating sump tank conditions for further applications at Zebrafish Core Facility, Endocrinology and Metabolism Research Institute (EMRI), Tehran University of Medical Sciences, Tehran, Iran. Fish were kept in aerated water with a pH around 7.5, oxygen content around $6.5 \pm 0.5 \mathrm{mg} / \mathrm{L}$, water temperature around $28 \pm 1^{\circ} \mathrm{C}$ and hardness of $250 \mathrm{mg} / \mathrm{L} \mathrm{CaCO3}$. Fish adjusted to a $14: 10$ hour light:dark cycle and water were replaced every other day [23]. The procedures used in this study adhered to the tenets of the Declaration of Helsinki. Random designated adult-bred males were transferred to specific incubators five days prior to experiments for acclimation. Fish fed with $20 \mathrm{mg} / \mathrm{fish}$ of commercial food containing $48 \%$ protein, $8 \%$ fat, and $2 \%$ fiber and freshly hatched Artemia every morning after light up. In this trial, 3 months old adult male fish were divided into four groups (two control group and two T2DM diabetic group) each one contained 15 fish. The experiments were performed in duplicate manner and duplicate grouping system. Each group was kept in 2-liter tanks under optimal conditions. The first healthy control group received no probiotic treatment $(\mathrm{HC})$, the second healthy control group treated with probiotic $(\mathrm{HC}-\mathrm{P})$, the first T2DM group received no probiotic supplement (T2DM) and the second T2DM group received probiotic supplements (T2DM-P). Diabetic condition was induced in both T2DM groups by gradient hyper-glucose accumulation methodology, starting from $50 \mathrm{mM}$ glucose concentration. Three days later, the glucose concentration raised to $100 \mathrm{mM}$, and at the end of the week it was increased to $200 \mathrm{mM}$. This gradual increasing of the glucose prevents fish fatality during conditioning period [24]. For biometric analysis, three fishes were randomly chosen from each group and allowed to reach a steady state within few minutes in a separate beaker and euthanized by Eugenol (Sigma-Aldrich, St. Louis, MO) using 3 drops in $50 \mathrm{ml}$ of RO water. Biometric parameters including fish length (from the head to the end of tail) and weight were measured and recorded and immediate blood glucose measurement was performed. 
Furthermore, body mass index (BMI) was measured based on dividing body weight (g) to the square of the body length $(\mathrm{cm})$.

\section{Probiotic administration}

Lactbacillus rhamnosus GG (ATCC: 53103 ) capsule was purchased from Culturelle Probiotics Co., Canada. To reach the adequate amount of probiotic consumption, final concentration of $10^{6}$ colonyforming units (CFU)/ml of the bacteria was dissolved in RO water, and used for treatments designated for $\mathrm{CP}$ and DP groups. The water in CP and DP tanks were gently replaced by fresh probiotic-rich water and used for incubation and further experimental steps.

\section{Blood glucose measurements}

To collect the blood, tail posterior was cut toward the anal with sterile seizure along with light pressure on tail, one drop of blood directly applied to strip on a commercial glucometer in duplicate manner, Match ${ }^{\text {TM }}$ (OK Biotech Co, Taiwan). Results were recorded and fish were then dissected to collect and preserve the intestinal tissue for histological analysis.

\section{Histological staining}

After collecting biometric results the same fish used for collecting intestinal sample. Intact intestine was removed and immediately stored in $10 \%$ formalin at $+4^{\circ} \mathrm{C}$. Following paraffin embedding, $10 \mu \mathrm{m}$ sections were prepared from the middle part of intestine and applied in hematoxylin and eosin (H\&E) as well as Albert's staining procedures $[25,26]$.

\section{Quantitative real-time polymerase chain reaction analysis}

To detect gene expression, total RNA was extracted from homogenized small intestine tissue using TRIzol reagent (Invitrogen, Carlsbad, CA). Reverse transcription was carried out with Gene Amp RNA PCR. Quantitative PCR (Applied Biosystems, Foster City, CA) was performed for each gene by a FluoCycle II TM Sybr Green master mix PCR kit using a standardized program ( $5^{\prime}$ initial denaturing step at $95^{\circ} \mathrm{C} ; 40$ cycles of $15^{\prime \prime}$ at $95^{\circ} \mathrm{C}, 20^{\prime \prime}$ at $55^{\circ} \mathrm{C}$, and $30^{\prime \prime}$ at $72^{\circ} \mathrm{C}$; melting point analysis in $0.1^{\circ} \mathrm{C}$ steps; final cooling step). All data were normalized to the expression of beta actin as housekeeping internal control gene. The primer sequences of IL-1 $\beta$ and TNF- $\alpha$ used for real-time PCR are summarized in table S1 (Supplementary Material). Relative quantification of target gene relative expression levels were calculated using $\Delta \Delta \mathrm{Ct}$ method [27].

\section{Statistical analysis}

Data are presented as mean \pm standard error. Student's t-test was used for comparison between the two experimental groups using the statistical software package SPSS Statistics version 16 (IBM Corp., Armonk, NY) with significance accepted at $p<0.001$.

\section{Results}


As summarized in Table S 2 (Supplementary Material), a significant decrease in the length of the diabetic group (T2DM) and probiotic-treated diabetics (T2DM-P) was observed compared to controls (HC). Moreover, we observed a slight increase in the length of the probiotic-treated controls (HC-P) $(P=0.013$,$) .$ Although both diabetic groups (T2DM and T2DM-P) showed lower weight compared to controls; the statistical analysis showed a significant difference between T2DM and HC $(P=0.0001)$. As shown in Table 2, a significant increase in the BMI of the HC-P group (1.255-fold) and a significant decrease in the T2DM group (0.343-fold) compared with HC $(p<0.05)$ was observed.

Impact of probiotics on blood glucose

We observed significant blood glucose elevation in the T2DM group compared with $\mathrm{HC}$ and $\mathrm{HC}-\mathrm{P}$ groups $(P<0.0001$, Fig. 1). Blood glucose has been decreased in the HC-P group in correlation with the supplemented probiotic $(P=0.011)$.

Influence of probiotics on zebrafish intestine

Histological analysis revealed some visible changes in the villus width and length during the probiotic treatment. Villus length has increased slightly in the T2DM-P group compared to T2DM, conversely, villus width has slightly increased in the T2DM group compared with the T2DM-P (Fig. 2). Histological analysis of the intestinal tissues indicated a wide hyperplasia in the goblet cells located in microvilli's of the T2DM and T2DM-P groups.

Influence of probiotics on zebrafish pro-inflammatory cytokine expression

The relative expression of pro-inflammatory cytokines, IL-1 $\beta$ and TNF- $\mathrm{a}$ was depicted in Fig. 3A and B. Compared to the control group, both IL-1 $\beta$ and TNF-a were over-expressed in the T2DM group $(P<$ 0.0001). The expression levels of IL-1 $\beta$ and TNF- $a$ increased by 2.75 and 3.77 -fold respectively, in the T2DM group compared with $\mathrm{HC}$. We found that probiotic supplementation resulted in a significant decrease in the expression levels of pro-inflammatory cytokines in T2DM-P group compare to T2DM $(P<$ 0.001).

Correlation between Blood Glucose and pro-inflammatory cytokine

The correlation between blood glucose in various groups of zebrafish and pro-inflammatory cytokines (IL$1 \beta$ and TNF-a) expression was $r=-0.966, p=0.001$ and $r=-0.984, p=0.001$, respectively (Fig. 3C \&D).

\section{Discussion}

A variety of genetic and feeding models of diabetes have been established, nevertheless, the majority of them have shown an inconsistency in pathological defects compared to human disease. Therefore, among creatures, zebrafish gained a growing platform for developmental research on diseases modeling, 
based on high similarity of digestive tract and comparable microbial and gut colonization with human [28-30]. Although, adult zebrafish has not been generally applied as an experimental model to study diabetes; here, we have well established it by overfeeding at adult stage, to study the effects of probiotic supplements on T2DM in vivo model.

To generate this model, we used a gradient hyper-glucose accumulation methodology. Physiologically, adult zebrafish absorb molecules from water to make a hyperosmotical internal environment, therefore, immersing them in a glucose containing solution (starting from $50 \mathrm{mM}$ ) and rising to final $200 \mathrm{mM}$ could be beneficial [31]. According to our findings, this protocol increases blood glucose up to $300 \mathrm{mg} / \mathrm{dL}$ in T2DM Zebrafish which is in accordance to Gleeson et al. who showed a up to $400 \mathrm{mg} / \mathrm{dL}$ increase in blood glucose of adult zebrafish immersed in a 1\% glucose solution [32]. Moreover, this method provides micro-environment stability and prevents fish fatality.

In the biometric result, probiotic-supplemented diet improved fish weight compared with the standard diet in both T2DM and $\mathrm{HC}$ groups similar to observations of Valcarce et al. [30]. Although, we did not expect to see significant changes on the length of the fish during the treatment periods, our data indicated a slight increase in the length and calculated body mass index (BMI) of HC-P group. This finding remarkably points out to the strong value of probiotic and contributes with the physiological conditions which enhances effective fish development and growth [14, 19].

Our histological analysis clearly showed some visible changes in the villus length and width during the probiotic treatment. Since the T2DM fish groups were under harsh condition due to high concentration of glucose, their homeostasis tries to adapt the condition to survive by extension of the villus diameter to increase the absorption. Additionally, result of intestinal staining specified hyperplasia in goblet cells located in microvillis' in the T2DM group. As, one of the primary characteristic sign of obesity is hyperplasia and hypertrophy; administration of probiotic had such an ability to prevent goblet cells disruption due to high glucose in digestion system of our zebrafish diabetic model $[33,34]$.

Alterations in the intestinal homoeostasis may play a major role in the development of systemic inflammatory diseases including diabetes $[35,36]$. One of the main challenges in T2DM patients is blood glucose management. In parallel with previous research, we showed that the consumption of probiotic based foods had significantly decreased blood glucose [37, 38]. Moreover, we identified the blood glucose level slightly lower in the HC-P group than the others. Since probiotic can strongly affect the growth, development and immune system improvement; thus, current results specified the probiotic Lactobacillus rhamnosus capability to improve tolerance in high glucose concentration [37].

Recently, the contribution of the mucosal immune system and the gut microbiome in metabolic disease including T2DM has been highly concerned [35, 39]. Our findings showed that relative mRNA expression levels of IL 1- $\beta$ and TNF- $\alpha$ were down-regulated in fish with probiotic supplementation despite the induction of innate immune-related cytokine genes by probiotic Chromobacterium aquaticum reported by Yi et al. [40]. This apparent inconsistency in cytokine profile was seen among the genus of Lactobacillus 
whereas $L$. sakei induced pro-inflammatory cytokines including IL-1 $\beta$ and TNF- $\alpha$; and $L$. johnsonii promoted the production of TGF- $\beta$ in cellular models [41].

Moreover, there was a robust positive correlation between both IL1- $\beta$ and TNF- $\alpha$ and blood glucose levels (Fig. 4C \& D). Delgadillo-Silva et al. showed that altered composition of the gut microbiome stimulate the intestinal residing innate and adaptive immune cells and induce a cytokine-mediated inflammation which is accompanied by hyperglycemia [42]. TNF as an inhibitor of insulin signaling is a major contributor towards obesity-related diseases. Indeed, it has been demonstrated that cytokines such as IL1- $\beta$ and TNFa may play role in the inflammatory destruction of insulin-producing $\beta$-cells in human T2DM [36, 43, 44]. Due to their synergistic effect, pharmacological blockage of mentioned cytokines has been clinically modulating inflammatory diseases; however, a therapeutic gap for managing islet inflammation and cytokine production in T2DM is present [45].

Finally, we assumed that probiotic bacteria, like L. rhamnosus, through their pathogen associated molecular pattern signaling pathway and bioactive components might reduce the immune-cell infiltration, decreased pro-inflammatory cytokines and ameliorate the hyperglycemic phenotype $\mathrm{n}$ fish models. Consumption of sufficient amount of probiotic could be a therapeutic goal for weight reduction to control T2DM [19]. We might highlighte these results as one of the valuable efficiency of probiotics not only in blood glucose management in T2DM patients but also as an advantage of probiotic supplement for overall health improvement

\section{Conclusion}

In summary, this study elucidates that probiotics such as Lactobacillus rhamnosus may hinder the blood glucose elevation in the T2DM group by their immunemodulatory effects. Thereby development of a probiotic based therapeutic formulation could promote healthiness in patients with T2DM.

\section{Limitations}

The limitation of this study includes lack of data on time dependent changes in blood glucose levels, cytokines levels and comparing different type of probiotics. Therefore, the effectiveness of various probiotic and their long-term impact on glucose levels should be investigated in this useful experimental model.

\section{Abbreviations}

ATCC: American Type Culture Collection;BMI: body mass index;BP: Base pair; CFU: colony forming unit;EMRI: Endocrinology and Metabolism Research Institute;HC: healthy control group received no probiotic treatment; HC-P: healthy control group treated with probiotic; H\&E: hematoxylin and eosin ; IL-1 $\beta$ : Interleukin 1 beta;Real-time PCR: real-time polymerase chain reaction SE: standard error;T2DM: type 2 
diabetes mellitus;T2DM: T2DM group received no probiotic supplement;T2DM-P: T2DM group received probiotic supplements; TGF- $\beta$ : Transforming growth factor beta; TNF-a: tumor necrosis factor alpha.

\section{Declarations}

\section{- Ethics approval and consent to participate}

All animal experiments were performed according to the ethical guidelines and standards of fish treating in Zebrafish Fish Core Facility (ethical code number: ZDB-LAB-190117-2).

\section{- Consent to publish}

Not applicable. As all images are entirely unidentifiable and there are no details on individuals reported within the manuscript, consent for publication of images may not be required

\section{- Availability of data and materials}

The data supporting our findings be presented within the manuscript and additional supporting files. Moreover, other datasets used during the current study are available from the corresponding author on reasonable request

\section{- Competing interests}

The authors declared no potential conflicts of interest with respect to the research, authorship, and/or publication of this article.

\section{- Funding}

The authors are thankful to Endocrinology and Metabolism Molecular-Cellular Sciences Institute, Tehran University of Medical Sciences (EMRI) to support this study (grant numbers 2129).

\section{- Authors' Contributions}

FB contributed during the experimental process, performed zebrafish diabetic modeling, animals tissue sampling and wrote the first manuscript. FS conducted probiotic dosing and control of treatments, performed statistical analysis and depictions and revised the manuscript. RF edited all drafts and rewrote the final version of the paper. HM provided special tools and reagents for animal husbandry and performed control of incubation conditions. RC performed statistical analysis and conducted probiotic dosing and control of treatments. FS consulted in special measures for animal husbandry and assisted in interpretation of histology data. MRK conceived, directed, and designed the study, obtained funding, edited all drafts and rewrote the final version of the paper. All authors read and approved the final manuscript

\section{- Acknowledgements}


The authors are thankful to Endocrinology and Metabolism Research Institute (EMRI), Tehran University of Medical Sciences to support this study (grant numbers 2129). We thank Mataleena Parikka, University of Tampere, for providing zebrafish husbandry information in this study.

\section{References}

1. Komaroff AL. The Microbiome and Risk for Obesity and Diabetes. Jama 2017; 317: 355-356 DOI: 10.1001/jama.2016.20099

2. Gong M, Wen S, Nguyen T et al. Converging Relationships of Obesity and Hyperuricemia with Special Reference to Metabolic Disorders and Plausible Therapeutic Implications. Diabetes, metabolic syndrome and obesity : targets and therapy 2020; 13: 943-962 DOI: 10.2147/DMSO.S232377

3. Manne ND, Ginjupalli GK, Rice KM et al. Long-term treatment with empagliflozin attenuates renal damage in obese zucker rat. Experimental and Clinical Endocrinology \& Diabetes 2020; 128: 512-519

4. Casazza K, Hanks LJ, Beasley TM et al. Beyond thriftiness: Independent and interactive effects of genetic and dietary factors on variations in fat deposition and distribution across populations. American journal of physical anthropology 2011; 145: 181-191 DOI: 10.1002/ajpa.21483

5. da Rocha Fernandes J, Ogurtsova K, Linnenkamp U et al. IDF Diabetes Atlas estimates of 2014 global health expenditures on diabetes. Diabetes research and clinical practice 2016; 117: 48-54 DOI: 10.1016/j.diabres.2016.04.016

6. Khalili L, Alipour B, Asghari Jafarabadi M et al. Probiotic assisted weight management as a main factor for glycemic control in patients with type 2 diabetes: a randomized controlled trial. Diabetology \& metabolic syndrome 2019; 11: 5 DOI: 10.1186/s13098-019-0400-7

7. Evans G, Wright D. Long-Term Evaluation of a UK Community Pharmacy-Based Weight Management Service. Pharmacy 2020; 8: DOI: 10.3390/pharmacy8010022

8. Yandrapalli S, Jolly G, Horblitt A et al. Cardiovascular Safety and Benefits of Non-Insulin Antihyperglycemic Drugs for the Treatment of Type 2 Diabetes Mellitus - Part 1. Cardiology in review 2020: DOI: $10.1097 / C R D .0000000000000308$

9. Forslund K, Hildebrand F, Nielsen T et al. Disentangling type 2 diabetes and metformin treatment signatures in the human gut microbiota. Nature 2015; 528: 262-266 DOI: 10.1038/nature15766

10. Barengolts E. Gut Microbiota, Prebiotics, Probiotics, and Synbiotics in Management of Obesity and Prediabetes: Review of Randomized Controlled Trials. Endocrine practice : official journal of the American College of Endocrinology and the American Association of Clinical Endocrinologists 2016; 22: 1224-1234 DOI: 10.4158/EP151157.RA

11. Barengolts E, Smith ED, Reutrakul S et al. The Effect of Probiotic Yogurt on Glycemic Control in Type 2 Diabetes or Obesity: A Meta-Analysis of Nine Randomized Controlled Trials. Nutrients 2019; 11: DOI: 10.3390/nu11030671

12. Atabati H, Esmaeili SA, Saburi E et al. Probiotics with ameliorating effects on the severity of skin inflammation in psoriasis: Evidence from experimental and clinical studies. Journal of cellular 
physiology 2020; 235: 8925-8937 DOI: 10.1002/jcp.29737

13. Yun SI, Park HO, Kang JH. Effect of Lactobacillus gasseri BNR17 on blood glucose levels and body weight in a mouse model of type 2 diabetes. Journal of applied microbiology 2009; 107: 1681-1686 DOI: 10.1111/j.1365-2672.2009.04350.x

14. Rad AH, Abbasalizadeh S, Vazifekhah $S$ et al. The Future of Diabetes Management by Healthy Probiotic Microorganisms. Current diabetes reviews 2017; 13: 582-589 DOI: 10.2174/1573399812666161014112515

15. Bayat A, Azizi-Soleiman F, Heidari-Beni M et al. Effect of Cucurbita ficifolia and Probiotic Yogurt Consumption on Blood Glucose, Lipid Profile, and Inflammatory Marker in Type 2 Diabetes. International journal of preventive medicine 2016; 7: 30 DOI: 10.4103/2008-7802.175455

16. Jalali SZ, shiri MR, Shirazi MG. Effect of Probiotics on Full Intestinal Feeding in Premature Infants: A Double Blind, Clinical Trial. Iranian Journal of Pediatrics 2020: e100139 DOI: 10.5812/ijp.100139

17. Tonucci LB, Olbrich Dos Santos KM, Licursi de Oliveira L et al. Clinical application of probiotics in type 2 diabetes mellitus: A randomized, double-blind, placebo-controlled study. Clinical nutrition 2017; 36: 85-92 DOI: 10.1016/j.clnu.2015.11.011

18. Aliashrafi $M$, Nasehi $M$, Zarrindast M-R et al. Association of microbiota-derived propionic acid and Alzheimer's disease; bioinformatics analysis. Journal of Diabetes \& Metabolic Disorders 2020: DOI: $10.1007 /$ s40200-020-00564-7

19. Kobyliak N, Conte C, Cammarota G et al. Probiotics in prevention and treatment of obesity: a critical view. Nutrition \& metabolism 2016; 13: 14 DOI: 10.1186/s12986-016-0067-0

20. Howe K, Clark MD, Torroja CF et al. The zebrafish reference genome sequence and its relationship to the human genome. Nature 2013; 496: 498-503 DOI: 10.1038/nature12111

21. Rendueles $\mathrm{O}$, Ferrieres L, Fretaud $\mathrm{M}$ et al. A new zebrafish model of oro-intestinal pathogen colonization reveals a key role for adhesion in protection by probiotic bacteria. PLoS pathogens 2012; 8: e1002815 DOI: 10.1371/journal.ppat.1002815

22. Shan $Y$, Fang $C$, Cheng $C$ et al. Immersion infection of germ-free zebrafish with Listeria monocytogenes induces transient expression of innate immune response genes. Frontiers in microbiology 2015; 6: 373 DOI: 10.3389/fmicb.2015.00373

23. Hedrera MI, Galdames JA, Jimenez-Reyes MF et al. Soybean meal induces intestinal inflammation in zebrafish larvae. PloS one 2013; 8: e69983 DOI: 10.1371/journal.pone.0069983

24. Khoshnevisan K, Baharifar H, Torabi F et al. Serotonin level as a potent diabetes biomarker based on electrochemical sensing: a new approach in a zebra fish model. Analytical and bioanalytical chemistry 2021: DOI: $10.1007 /$ s00216-020-03122-5

25. Chan JK. The wonderful colors of the hematoxylin-eosin stain in diagnostic surgical pathology. International journal of surgical pathology 2014; 22: 12-32 DOI: 10.1177/1066896913517939

26. Wittekind $D$. Traditional staining for routine diagnostic pathology including the role of tannic acid. 1 . Value and limitations of the hematoxylin-eosin stain. Biotechnic \& histochemistry : official 
publication of the Biological Stain Commission 2003; 78: 261-270 DOl:

10.1080/10520290310001633725

27. Livak KJ, Schmittgen TD. Analysis of relative gene expression data using real-time quantitative PCR and the 2(-Delta Delta C(T)) Method. Methods 2001; 25: 402-408 DOI: 10.1006/meth.2001.1262

28. Zang L, Maddison LA, Chen W. Zebrafish as a Model for Obesity and Diabetes. Frontiers in cell and developmental biology 2018; 6: 91 DOI: 10.3389/fcell.2018.00091

29. Goldsmith JR, Jobin C. Think small: zebrafish as a model system of human pathology. Journal of biomedicine \& biotechnology 2012; 2012: 817341 DOI: 10.1155/2012/817341

30. Valcarce DG, Riesco MF, Martinez-Vazquez JM et al. Diet Supplemented with Antioxidant and AntiInflammatory Probiotics Improves Sperm Quality after Only One Spermatogenic Cycle in Zebrafish Model. Nutrients 2019; 11: DOI: 10.3390/nu11040843

31. Capiotti KM, Antonioli R, Jr., Kist LW et al. Persistent impaired glucose metabolism in a zebrafish hyperglycemia model. Comparative biochemistry and physiology Part B, Biochemistry \& molecular biology 2014; 171: 58-65 DOI: 10.1016/j.cbpb.2014.03.005

32. Gleeson M, Connaughton V, Arneson LS. Induction of hyperglycaemia in zebrafish (Danio rerio) leads to morphological changes in the retina. Acta diabetologica 2007; 44: 157-163 DOI: 10.1007/s00592007-0257-3

33. Flynn EJ, 3rd, Trent CM, Rawls JF. Ontogeny and nutritional control of adipogenesis in zebrafish (Danio rerio). Journal of lipid research 2009; 50: 1641-1652 DOI: 10.1194/jlr.M800590-JLR200

34. Subbotin VM. Analysis of arterial intimal hyperplasia: review and hypothesis. Theoretical biology \& medical modelling 2007; 4: 41 DOI: 10.1186/1742-4682-4-41

35. Winer DA, Luck H, Tsai S et al. The Intestinal Immune System in Obesity and Insulin Resistance. Cell metabolism 2016; 23: 413-426 DOI: 10.1016/j.cmet.2016.01.003

36. Hernandez-Santana YE, Giannoudaki E, Leon G et al. Current perspectives on the interleukin-1 family as targets for inflammatory disease. European journal of immunology 2019; 49: 1306-1320 DOI: 10.1002/eji.201848056

37. Yao K, Zeng L, He Q et al. Effect of Probiotics on Glucose and Lipid Metabolism in Type 2 Diabetes Mellitus: A Meta-Analysis of 12 Randomized Controlled Trials. Medical science monitor: international medical journal of experimental and clinical research 2017; 23: 3044-3053 DOI: $10.12659 / \mathrm{msm} .902600$

38. Kobyliak N, Falalyeyeva T, Mykhalchyshyn G et al. Effect of alive probiotic on insulin resistance in type 2 diabetes patients: Randomized clinical trial. Diabetes \& metabolic syndrome 2018; $12: 617-$ 624 DOI: 10.1016/j.dsx.2018.04.015

39. Zeinali F, Aghaei Zarch SM, Vahidi Mehrjardi MY et al. Effects of synbiotic supplementation on gut microbiome, serum level of TNF-alpha, and expression of microRNA-126 and microRNA-146a in patients with type 2 diabetes mellitus: study protocol for a double-blind controlled randomized clinical trial. Trials 2020; 21: 324 DOI: 10.1186/s13063-020-04236-y 
40. Yi CC, Liu $\mathrm{CH}$, Chuang KP et al. A potential probiotic Chromobacterium aquaticum with bacteriocinlike activity enhances the expression of indicator genes associated with nutrient metabolism, growth performance and innate immunity against pathogen infections in zebrafish (Danio rerio). Fish \& shellfish immunology 2019; 93: 124-134 DOI: 10.1016/j.fsi.2019.07.042

41. Azad MAK, Sarker M, Wan D. Immunomodulatory Effects of Probiotics on Cytokine Profiles. BioMed research international 2018; 2018: 8063647 DOI: 10.1155/2018/8063647

42. Delgadillo-Silva LF, Tsakmaki A, Akhtar $\mathrm{N}$ et al. Modelling pancreatic beta-cell inflammation in zebrafish identifies the natural product wedelolactone for human islet protection. Disease models \& mechanisms 2019; 12: DOI: 10.1242/dmm.036004

43. Tsarouchas $T M$, Wehner $D$, Cavone $L$ et al. Dynamic control of proinflammatory cytokines II-1 beta and Tnf-alpha by macrophages in zebrafish spinal cord regeneration. Nature communications 2018; 9: 4670 DOI: 10.1038/s41467-018-07036-w

44. Wang G, Liang R, Liu T et al. Opposing effects of IL-1beta/COX-2/PGE2 pathway loop on islets in type 2 diabetes mellitus. Endocrine journal 2019; 66: 691-699 DOI: 10.1507/endocrj.EJ19-0015

45. Nguyen T, Payan B, Zambrano A et al. Epigallocatechin-3-gallate suppresses neutrophil migration speed in a transgenic zebrafish model accompanied by reduced inflammatory mediators. Journal of inflammation research 2019; 12: 231-239 DOI: 10.2147/JIR.S224834

\section{Figure}

Figure 4 not available with this version.

\section{Figures}




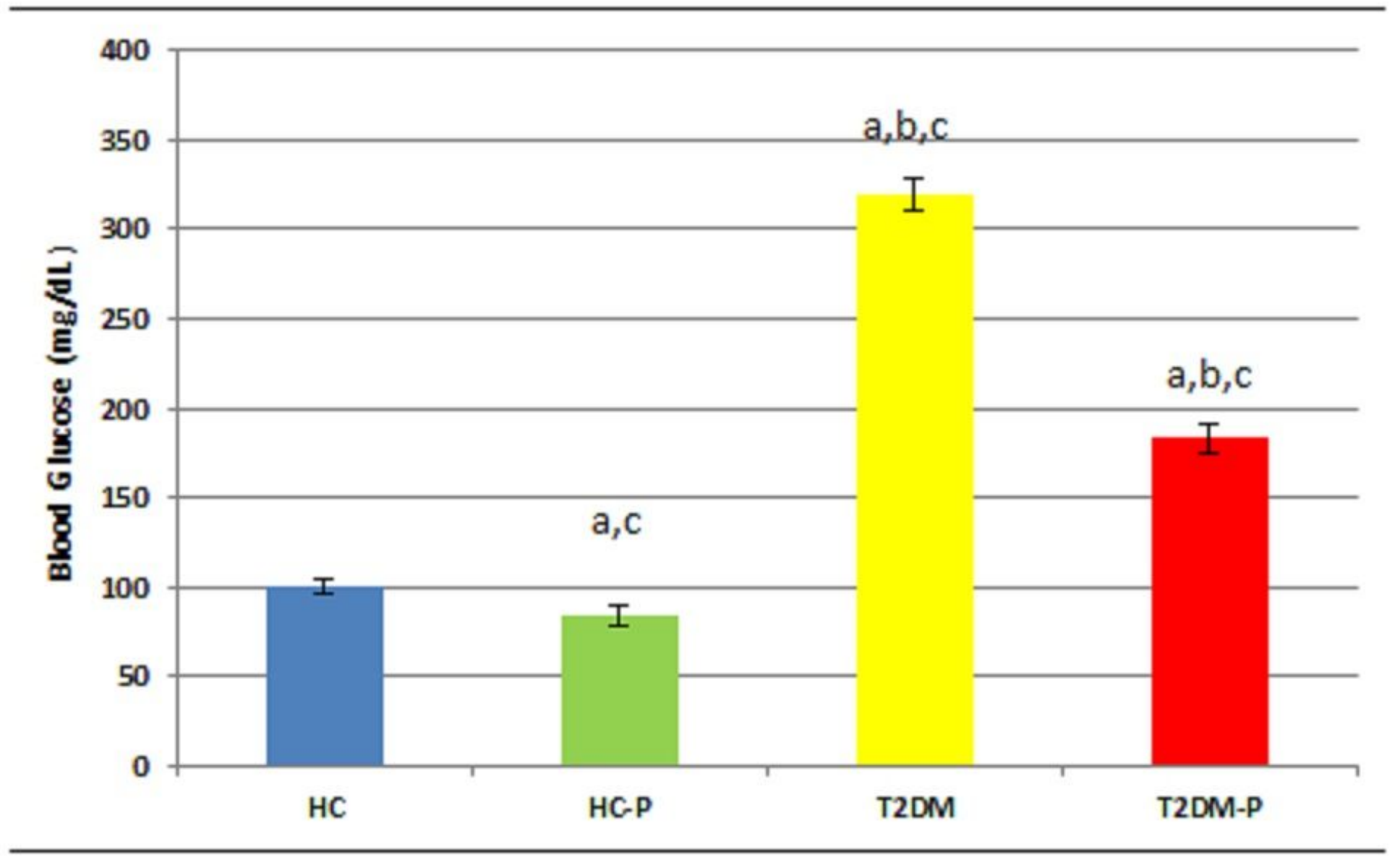

\section{Figure 1}

This diagram represents the result of blood glucose from all trail groups. Data represents the mean+ SE of the results for three independent assays. Statistical analysis compares difference of the mean of results between T2DM-P and HC, HC-P and T2DM. Difference with $p<0.001$ was considered significant. Each group contained 6 zebrafish. 

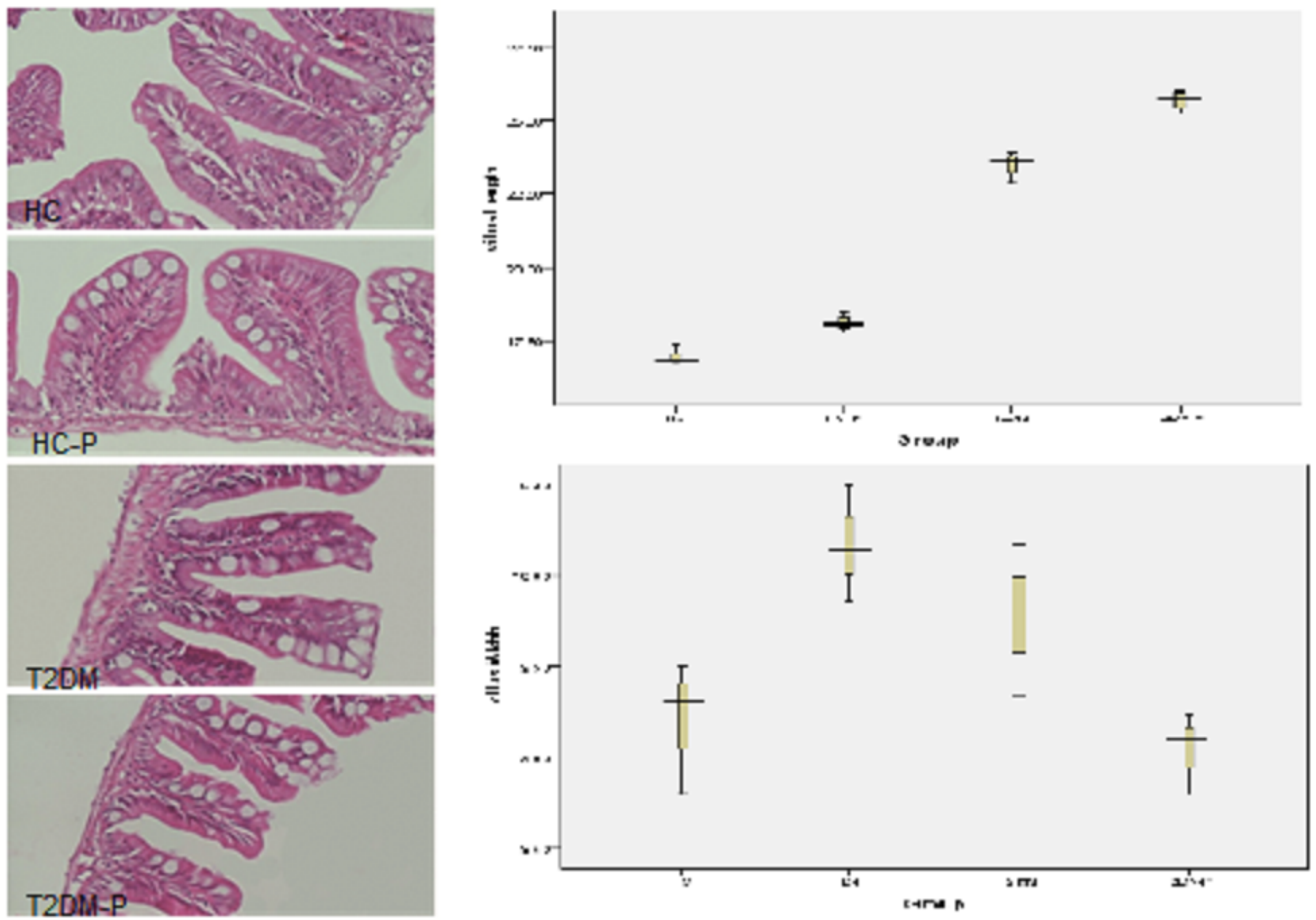

Figure 2

Histopathology evaluation of zebrafish small intestine. Intestinal tissues were stained with H\&E and studied by microscopy ( $\times 400$ resolution). Healthy control group $(\mathrm{HC})$, Healthy control group supplemented with probiotics (HC-P), Diabetic group (T2DM), Diabetic group supplemented with probiotic (T2DM-P). (A) Villus length increased slightly in the T2DM-P group following probiotic supplementation compared to T2DM group. (B). Villus width was slightly higher in the T2DM group compared to T2DM-P group. Data shows mean + SE for three independent assays. 

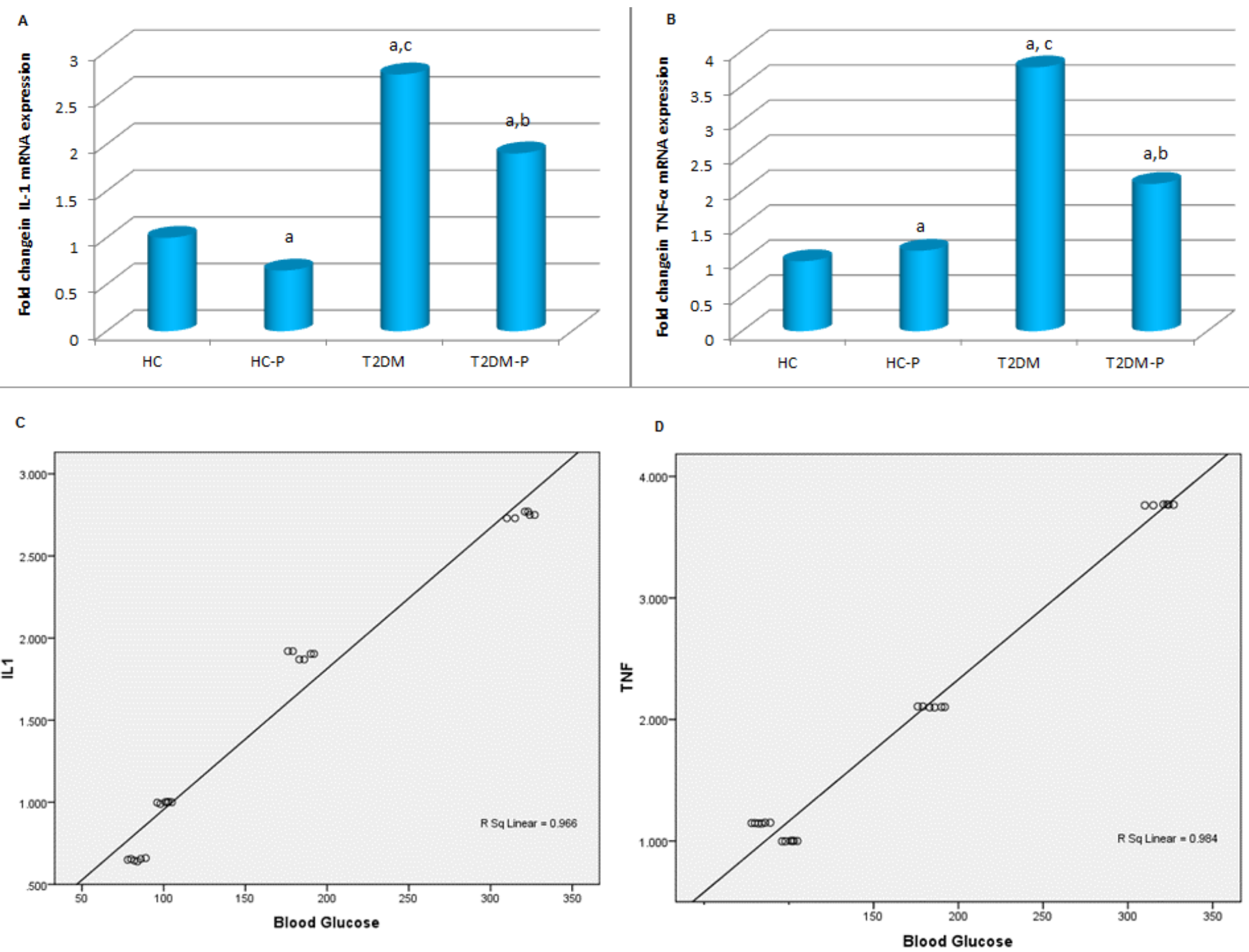

Figure 3

The relative expression of the intestinal pro-inflammatory cytokines. A) The effects of probiotic on mRNA expression of IL-1 $\beta$ were determined by PCR. B) The relative expression of TNF- $a$ from all trail groups represent. The expression levels of $\beta$-actin were used as a loading control. C) The Correlation of IL-1 $\beta$ to blood glucose; D) The Correlation of TNF-a vs. blood glucose. Control group(HC), Control + Probiotic (HC$P)$, Diabetic group (T2DM) , Diabetic group with probiotic(T2DM-P) groups. a Significance vs. HC; $b$ Significance vs. HC-P; c Significance vs. T2DM-P, The mean difference is significant at $p<0.001$.

\section{Supplementary Files}

This is a list of supplementary files associated with this preprint. Click to download.

- TableS1.docx

- Tables2.docx 